\title{
Optimal microgrid planning for enhancing ancillary service provision
}

\author{
Sergio F. CONTRERAS ${ }^{1}$, Camilo A. CORTES $^{2}$ (D, Johanna M. A. MYRZIK ${ }^{1}$
}

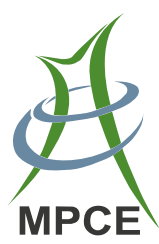

\begin{abstract}
Microgrids have presented themselves as an effective concept to guarantee a reliable, efficient and sustainable electricity delivery during the current transition era from passive to active distribution networks. Moreover, microgrids could offer effective ancillary services (AS) to the power utility, although this will not be possible before the traditional planning and operation methodologies are updated. Hence, a probabilistic multi-objective microgrid planning (POMMP) methodology is proposed in this paper to contemplate the large number of variables, multiple objectives, and different constraints and uncertainties involved in the microgrid planning. The planning methodology is based on the optimal size and location of energy distributed resources with the goal of minimizing the mismatch power in islanded mode, while the residual power for AS provision and the investment and operation costs of the microgrid in grid-connected mode are
\end{abstract}

CrossCheck date: 13 February 2019

Received: 17 November 2018/Accepted: 13 February 2019/ Published online: 3 June 2019

(C) The Author(s) 2019

$\triangle$ Camilo A. CORTES

caacortesgu@unal.edu.co

Sergio F. CONTRERAS

ContrerasS@iat.uni-bremen.de

Johanna M. A. MYRZIK

MyrzikJ@iat.uni-bremen.de

1 Institute of Automation Technology, University of Bremen, Otto-Hahn-Allee 1 NW1/M1050(70), 28359 Bremen, Germany

2 Department of Electrical and Electronics Engineering, Universidad Nacional de Colombia, Carrera 30 Calle 45. Ciudad Universitaria Edificio 453, Bogotá, Colombia maximized and minimized, respectively. For that purpose, probabilistic models and a true multi-objective optimization problem are implemented in the methodology. The methodology is tested in an adapted PG\&E 69-bus distribution system and the non-dominated sorting genetic algorithm II (NSGA-II) optimization method and an analytic hierarchy process for decision-making are used to solve the optimization problem.

Keywords Microgrid, Ancillary services (AS), Planning, Multi-objective optimization, Decision-making

\section{Introduction}

Microgrids are active medium- and low-voltage distribution networks that can operate in either grid-connected or islanded modes by means of the operation of interconnected distributed energy resources and different loads $[1,2]$. In last decades, the concept of microgrid has drawn an special attention from academics and stakeholders in order to not only manage the transition from passive to active distribution networks but also improve the reliability, security, and efficiency of the main utility grids through the provision of ancillary services (AS) by the microgrids [3]. In fact, a microgrid might be considered as a flexible self-controlled entity that can be seen as an aggregated load or power source by the main grid operators, which gives rise to a valuable operational resource to the main grid [2]. The implementation of the concept of microgrid leads to numerous technical and regulatory issues that should be carefully tackled before the design and planning.

In this direction, many models and methods have been proposed to solve the active distribution network planning problem in the last decades. For example, [4] and [5] 
present a complete review of proposed models and optimization approaches for the optimal planning of active distribution networks. Reference [6] focuses on the computational optimization techniques that are used particularly for the microgrid planning. It can be remarked from the conclusion that there are still microgrid planning aspects without adequate or acute consideration.

For example, despite the plausible capacity of microgrids to provide AS to the main utility system and participate in the electricity market [7], the AS capacity has been commonly left outside of the planning problem [3]. On the other hand, although the penetration of distributed energy resources with stochastic behaviour gives rise to a planning with uncertainties, it has not been widely studied in the microgrids case $[4,5]$. Furthermore, although the grid-connected and islanded operation modes are relevant characteristics of the microgrid's flexible operation, these are normally considered as separated problems [6].

Moreover, [6] shows that despite the microgrid planning problem involves several conflictive objectives, most of the research around is based on mono-objective optimization strategies. In fact, there are research that considers more than one objective function, but the set of objective functions are normally transformed into a virtual monoobjective function [8]. For instance, [9, 10] propose a probabilistic model to optimally adequate a distribution network with distributed generation (DG) and distributed storage (DS), but the methodology with two objective functions are solved by means of a virtual mono-objective one. Reference [8] presents a complete review of the multiobjective planning of distributed energy resources, and claim that planning optimization problems should be formulated and solved as true-multi-objective strategies due to their involved complexity. This assertion is reinforced in [11], where it is solved as a distribution network planning problem by means of a probabilistic-based DG model and true-multi-objective strategy. Thus, results in [11] support the multi-objective strategy convenience, although in this work the DS penetration, the islanded mode operation, and an AS capability are not contemplated.

References [12,13] present a multi-objective stochastic chance-constrained programming model for the planning of stand-alone microgrids. In the first one, the net present cost in the life cycle and pollutant emissions are minimized. In the second one, the generation cost for distribution companies is minimized and an internal return rate for DG owners is maximized. However, despite both works highlight the advantages of a multi-objective strategy, the research is limited to an stand-alone operation, and the AS are not considered as part of the planning problem. Moreover, a decision-making strategy over the Pareto-front to choose the optimal microgrid design from the outcome is not implemented either.
Regarding the AS provision by microgrids, [7] presents for the first time an assessment of the technical feasibility of microgrids to supply AS, which is also amply discussed in [2]. Nonetheless, most research has been focused on the operation problem, AS supplying viability, and electricity market conditioning for the microgrid AS participation [14-17]. For example, in [18] a system-level assessment framework has been recently proposed for evaluating the contribution of microgrids in the power system capacity planning and the development of policies for AS markets. Reference [19] develops a microgrid planning model to simulate the optimal operation of the microgrid and calculate the system cash flow. This model is used to design a campus microgrid. The reviewed research demonstrates and highlights the potential of microgrids in the provision of AS, but they do not completely tackle the technical considerations from the perspective of microgrid planning.

A modification of the distributed energy resources customer adoption model (DER-CAM) is proposed in [3] with the proposal of including the stream revenue from the participation of a microgrid in the AS market. The DERCAM is a mathematical model developed to determine different types and capacities of distributed energy resources inside of a microgrid and find the optimal dispatch of them [3]. The results presented in [3] suggest that the participation of microgrids in AS markets can affect their optimal sizing of resources as well as the dispatch of energy. Nevertheless, [3] proposes a deterministic model based on a mono-objective cost function, which has been found as a drawback due to the actual presence of more than one contradictory objective that cannot be modelled accurately as a priory in the planning problem [11].

In [1], a probabilistic multi-objective microgrid planning (POMMP) methodology is proposed to find the optimal locations and capacities of distributed energy resources considering three objective functions: the minimization of the power mismatch in islanded mode; the maximization of the residual active power to be exported to the main network; and the minimization of the annual energy losses in grid-connected mode. In this case, the planning methodology focuses on the technical optimization of AS supplying instead of the economic issues, which is a shortcoming of the research. Nonetheless, the research shows the advantages of the methodology to solve the microgrid planning problem.

This paper is the extension of the work in [1], and a POMMP methodology with technical and economical objective functions for the expansion or transformation of existing active or passive distribution networks under the concept of microgrids with AS capacity is proposed. 
The POMMP methodology enhances the proposal in [1] by modifying both the microgrid's mathematical model and the planning optimization problem as well as including a decision-making strategy to select the optimal solution from the Pareto-front outcome. Thus, the main contributions of the paper are as follows.

1) The vector of decision variables is modified to enhance the sizing and location of DS resources. Furthermore, the charging/discharging (C/D) cycles and operation criteria of DS have been modified based on the AS provision and a demand management approach.

2) An economic objective function to minimize the operation cost of the microgrid is introduced, which lead to a multi-objective optimization problem with three objective functions: maximization of the active power in grid-connected mode; minimization of the mismatch active power in islanded mode and the minimization of the operating cost of the microgrid.

3) The set of constraint functions is enhanced to improve the reliability of methodology.

4) A decision-making stage based on the analytic hierarchy process (AHP) technique is implemented to properly choose an optimal microgrid design from the Pareto-set that is obtained with the non-dominated sorting genetic algorithm II (NSGA-II) approach. This decision-making technique is carefully chosen based on the advantages reported in [20], and the analysis of other methods such as the simple additive weighting, the technique for order of preference by similarity to ideal solution, and the multi-attribute utility theory (MAUT).

Regarding the AS provision, the proposed POMMP methodology optimally plans the microgrid to have an AS capability based on a fully available extra active power capacity to be exported back to the main grid and to provide up- and down-frequency regulation, and spinning and non-spinning operating reserve $[3,21]$.

This paper is divided in seven sections. In Section 2, the microgrid mathematical model is presented. The optimization problem is formulated in Section 3 and the planning methodology is proposed in Section 4. In Section 5, the test system Pacific Gas \& Electric (PG\&E) 69-bus, simulation conditions and two modelled cases based on two market scenarios: california independent system operator (CAISO) and PJM Interconnection LLC (PJM) are explained. In Section 6, the optimal planning results are found and analyzed using the NSGA-II and AHP. Finally, the conclusions of the research are presented.

\section{Microgrid mathematical model}

In this section, the microgrid mathematical models for the POMMP methodology are explained.

\subsection{Model of dispatchable and non-dispatchable units}

For the POMMP methodology, the microgrid is considered with dispatchable and non-dispatched DG units such as photovoltaic (PV) modules, wind turbines (WT) and microturbines (MT). The PV units $c$ are considered for the planning as continuos generation technologies, while the WT and MT units $g$ are modeled as discrete generation technologies. The set of DG technologies $(c \cup g)$ is denoted by $j$. The DG models are defined based on [1] as:

1) Non-dispatchable DG: PV and WT technologies are modelled as non-dispatchable units due to their stochastic operation. Thus, the available active power is calculated with the probability density function (PDF) of the primary energies (wind speed and solar irradiation). The PDFs are build from historical timeseries following the procedure in [1] and [3] along the planning horizon. The uncertainty in the planning due to the stochastic performance of the generation units is considered through Monte Carlo simulation of the primary energy, which is generated based on the PDF parameters. Afterwards, the wind speed and solar irradiation value with the biggest probability during $t$ are chosen to calculate the available active power. The transformation functions to calculate the available active power of WT and PV are shown in (1) and (2), respectively [1].

$P a_{w t}= \begin{cases}0 & v<v_{i} \text { and } v>v_{o} \\ P r_{w t} \frac{v-v_{i}}{v_{r}-v_{i}} & v_{i} \leq v<v_{r} \\ P r_{w t} & v_{r} \leq v \leq v_{o}\end{cases}$

where $P a_{w t}$ is the available active power output; $P r_{w t}$ is the rated power; and $v_{i}, v_{r}, v_{o}$ are the cut-in, rated and cut-out wind speeds, respectively.

$P a_{p v}= \begin{cases}\operatorname{Pr}_{p v} \frac{G^{2}}{G_{s t c} R_{c}} & 0 \leq G<R_{c} \\ \operatorname{Pr}_{p v} \frac{G}{G_{s t c}} & R_{c} \leq G \leq G_{s t c} \\ \operatorname{Pr}_{p v} & G>G_{s t c}\end{cases}$

where $P a_{p v}$ is the available active power output; $P r_{p v}$ is the equivalent rated power; $G$ is the solar irradiance; $G_{s t c}$ is the solar irradiance in standar test conditions of $1000 \mathrm{~W} / \mathrm{m}^{2}$; and $R_{c}$ is an specific irradiation point that accordingly to [22] is typically $150 \mathrm{~W} / \mathrm{m}^{2}$. 
2) Dispatchable DG generation: MTs are modelled as dispatchable generators without uncertainty. Therefore, MTs can generate a specified output power with a certain power factor during the planning horizon [1].

\subsection{Model of energy storage systems}

The POMMP methodology can be adapted for different technologies of energy storage systems. Nevertheless, it has been modelled as a set of lead-acid batteries (BA) as in [1]. For the model proposed in this paper, binary decision variables to simulate the operation control of the $C / D$ cycle of DS are introduced. Hence, in grid-connected operation mode, the binary operation variables $b_{R D n_{m, d, h}}$, for selling frequency down-regulation, and $b_{A u x_{m, d, h}}$, for supplying AS, are used $[1,3]$. Furthermore, C/D cycles are commanded by a demand management strategy based on the power demand-generation rate of microgrid in grid-connected operation mode and islanded operation mode. This is shown in (3)-(8), where for the battery $k, S O C_{k}$ is the battery state of charge; $M S C_{k}$ is the maximum charge; $D O D_{k}$ is the minimum deep of charge; $\eta_{b a_{k}}$ is the battery efficiency; $P_{b a_{k}}$ is the available battery active power; and $C_{b a_{k}}$ is the battery capacity.

Subscripts $m, d$ and $h$ describe a time-segment $t$ based on a month, day, and hour condition along the planning horizon.

$$
\begin{aligned}
& \operatorname{SOC}_{k}(t+1)=S O C_{k}(t)\left(1-\eta_{b a_{k}}\right) \pm \frac{P_{b a_{k}}}{C_{b a_{k}}} \\
& P_{b a_{k}}\left(S O C_{k}\right)=\left\{\begin{array}{cc}
P_{b a_{k}}^{c h} \eta_{c h_{k}} & b_{c h_{m, d, h}}=1 \\
P_{b a_{k}}^{d c h} / \eta_{d c h_{k}} & b_{d c h_{m, d, h}}=1
\end{array}\right.
\end{aligned}
$$

where $P_{b a_{k}}^{c h}, P_{b a_{k}}^{d c h}$ are the battery $k \mathrm{C} / \mathrm{D}$ power, respectively; and $\eta_{c h_{k}}, \eta_{d c h_{k}}$ are the battery $k$ input/output efficiency, respectively.

The binary variables for commanding the C/D cycles are defined as:

1) Grid-connected mode:

$$
\begin{aligned}
& b_{c h_{m, d, h}}=1 S O C \leq M S C, b_{R D n_{m, d, h}}=1 \text { or } b_{B p k_{m, d, h}}=1 \\
& b_{d c h_{m, d, h}}=1 S O C \geq D O D, b_{A u x_{m, d, h}}=1 \text { or } b_{B p k_{m, d, h}}=0
\end{aligned}
$$

2) Islanded mode:

$b_{c h_{m, d, h}}=1 S O C \leq M S C, b_{B p k_{m, d, h}}=1$

$b_{d c h_{m, d, h}}=1 S O C \geq D O D, b_{B p k_{m, d, h}}=0$

where $b_{B p k_{m, d, h}}$ is defined in (9).

$$
b_{B p k_{m, d, h}}=\left\{\begin{array}{cc}
1 & P_{D G_{m, d, h}}-\left(P_{\text {load }_{m, d, h}}+P_{\text {loss }_{m, d, h}}\right)>0 \\
& \text { or } P_{\text {load }_{m, d, h}} \leq 1.2 P_{\text {load }}^{\text {min }} \\
& \\
0 & P_{D G_{m, d, h}}-\left(P_{\text {load }_{m, d, h}}+P_{\text {loss }_{m, d, h}}\right) \leq 0 \\
& \text { or } P_{\text {load }_{m, d, h}} \leq 0.8 P_{\text {load }}^{\text {max }}
\end{array}\right.
$$

where $P_{D G_{m, d, h}}$ is the active power generated by the DG; $P_{\text {load }_{m, d, h}}$ is the active power demanded by the load; and $P_{\text {loss }_{m, d, h}}$ are the power losses. $b_{R D n_{m, d, h}}$ and $b_{A u x_{m, d, h}}$ change their state depending on the market and the main network operator simulation proposed and explained later.

\subsection{Loads profiles and planning horizon}

In [3], a time distribution with three different typical type of days: weekdays (ES), weekends (FS) and peak days (DP), is used during the planning horizon. Thus, for the model proposed in this paper, a planning horizon of one year with these three typical days per month and time segments of one hour per day is considered. The load profile for each typical day is found from historical time series $[3,23]$.

\subsection{Microgrid operation modes and network operator simulation strategies}

The POMMP methodology considers objective functions and operation conditions under grid-connected and islanded modes. The strategy implemented in [1] for considering the islanded operation is implemented in POMMP. On the other hand, there is an inherent difficulty to accurately forecast the network operator request of AS during the horizon planning, which introduces uncertainties in the planning process. Therefore, in POMMP an strategy to consider the AS provision to the main utility grid is proposed. This consists in comparing the clearing prices of each AS type (up- and down-frequency regulation, spinning reserve and non-spinning reserve) from historical time series, and the AS with the highest clearing price is chosen to economically optimize the revenue stream of microgrid. The strategy is modelled in (10).

$$
\begin{aligned}
& T_{a r_{m, d, h}}^{\max }=\max ( T_{E x_{m, d, h}}, S_{M C P_{m, d, h}}, \\
&\left.N S_{M C P_{m, d, h}}, R_{U p M C P_{m, d, h}}, R_{D n M C P_{m, d, h}}\right)
\end{aligned}
$$

where $T_{a r_{m, d} h}^{\max }$ is the maximum rate between different services to be exported to the grid; $T_{E x_{m, d, h}}$ is the regulated tariff for electricity; and $S_{M C P_{m, d, h}}, N S_{M C P_{m, d, h}}, R_{U p M C P_{m, d, h}}$, $R_{D n M C P_{m, d, h}}$ are the regulated tariffs for spinning reserve, non-spinning reserve, and up- and down-frequency regulation, respectively. Afterwards, binary values are assigned to the associated binary variables of each one of the 
possible AS markets in which the microgrid optimally could participate along the planning horizon. Then, $b_{T E x_{m, d, h}}$ is the binary decision of selling electricity to the stock, and $b_{S_{m, d, l}}, b_{N S_{m, d, h}}, b_{R U p_{m, d, h}}$, and $b_{R D n_{m, d, h}}$ are the binary decision of selling certain type of AS respectively (spinning reserve, non-spinning reserve, and up- and down-frequency regulation).

\subsubsection{Key aspects}

1) It is important to consider that each territory has its own market rules with different types of energy dispatch and different regulations [3].

2) The microgrid is seen by the main utility grid operator as a generation or load bus that can transfer or consume electrical energy. Hence, the AS supply is based on the active power that can be generated or consumed in the microgrid.

3) The microgrid will be price taker having the clearing prices in the electricity markets, which it is supposed to be a proper bid strategy to compete in an open market [3].

4) The clearing prices of each type of AS represent the requirements of the electricity market to maintain the reliability and safety of the power system [3].

\section{Multi-objective optimization problem formulation of microgrid planning}

A true-multi-objective optimization model is formed by the decision variables vector, objective functions, constraint functions, and the mathematical model. Furthermore, the problem is solved in three parts: model setup, optimization, and decision-making [24]. The POMMP methodology is proposed as a true-multi-objective optimization problem with three objective functions, $N_{d}$ decision variables, and $N_{c}$ constraints functions to form the feasible region as it is described below. The optimization problem is solved with the NSGA-II, while the chosen optimal solution is selected using the AHP multicriteria decision-making technique.

\subsection{Objective functions for enhanced POMMP methodolgy}

One of the contributions of this paper is the enhanced optimization problem for the planning of microgrids with AS capacity. In this proposal, the residual active power in grid-connected mode is maximized, the power mismatch in islanded mode is minimized, and the operation cost in gridconnected is minimized. Consider $j$ for DG units, $k$ for DS units, and $l$ for loads. The set of distributed energy resources $(j \cup k)$ is denoted by $i$. Furthermore, consider the variables introduced in Section 2.2 for describing the objective functions $f_{1}$ and $f_{2}$.

\subsubsection{Microgrid residual power in grid-connected mode}

The residual active power in grid-connected mode is calculated in (11) for $N_{t}$ time segments $t$.

$$
\begin{gathered}
f_{1}(x)=-\frac{1}{N_{t}}\left[\sum _ { t = 1 } ^ { N _ { t } } \left(\sum_{j} P_{D G_{j, t}}(x) \pm \sum_{k} P_{b a_{k, t}}(x)\right.\right. \\
\left.\left.-\sum_{l} P_{\text {load }_{l, t}}(x)-P_{\text {loss }_{t}}(x)\right)\right] \quad \forall t
\end{gathered}
$$

\subsubsection{Microgrid active power mismatch in islanded mode}

The objective function is given in (12).

$$
\begin{gathered}
f_{2}(x)=\frac{1}{N_{t}}\left[\sum _ { t = 1 } ^ { N _ { t } } \left(\sum_{j} P_{D G_{j, t}}(x) \pm \sum_{k} P_{b a_{k, t}}(x)\right.\right. \\
\left.\left.-\sum_{l_{\text {sched }}} P_{\text {lshed }_{\text {sched }},}(x)-P_{\text {Iloss }}(x)\right)\right] \quad \forall t
\end{gathered}
$$

where $P_{\text {lshed }}$ is the power demanded by the loads $l_{\text {shed }}$ after a load shedding as a possible demand managing strategy in islanded mode operation.

\subsubsection{Minimization of microgrid operation cost in grid- connected mode}

In POMMP, an economic function is introduced to enhanced the proposal in [1]. Its objective is to minimize the operation and investment costs of a microgrid in a typical year. The main considerations are as follows.

1) Customer's loads are modelled accordingly to the planning horizon.

2) Investment costs are annualized based on an interest rate and the lifespan of the technology.

3) The installed capacity of PV and BA technologies is sized using continuous decision variables while the capacity of the MT and WT technologies is sized through discrete decision variables [3].

The objective function in (13) contains a set of desired components of costs to be optimally minimized in the microgrid in a year, including the regulated tariff for public services, the energy purchasing cost, the operating and maintenance costs (O\&M) for the on-site generation, the annualized capital costs of the distributed energy resources, the revenues from sales of electricity and the provision of AS to the grid. 


$$
\begin{aligned}
f_{3}(x) & \\
= & \sum_{m} T_{F_{m}} \\
& +\sum_{m} \sum_{d} \sum_{h} U_{L_{m, d, h}} T_{E_{m, d, h}} \\
& +\sum_{j} \sum_{m} \sum_{d} \sum_{h} G_{T_{j, m, d, h}}\left(C_{V C_{j}}+C_{O M V_{j}}\right) \\
& +\sum_{g} I_{G_{g}} \max \left(P_{g}\left(C_{C C D_{g}} A_{g}+C_{O M F_{g}}\right)\right) \\
& +\sum_{c}\left(C_{F C C_{c}} \operatorname{Cap}_{c}\right) A_{c}+C_{a p_{c}} C_{O M F_{c}} \\
& \left.+\sum_{k}\left(C_{F C C_{k}} \operatorname{Cap}_{k}\right) A_{k}+C_{a p_{k}} C_{O M F_{k}}\right) \\
& -\sum_{m} \sum_{d} \sum_{h} G_{S_{m, d, h}} T_{E x_{s, d, h}} b_{T E x_{m, d, h}} \\
& -\sum_{m} \sum_{d} \sum_{h} G_{S_{m, d, h}} S_{M C P_{m, d, h}} b_{S_{m, d, h}} \\
& -\sum_{m} \sum_{d} \sum_{h} G_{S_{m, d, h}} N S_{M C P_{m, d, h}} b_{N S_{m, d, h}} \\
& -\sum_{m} \sum_{d} \sum_{h} G_{S_{m, d, h}} R_{U p M C P_{m, d, h}} b_{R U p_{m, d, h}} \\
& -\sum_{m} \sum_{d} \sum_{h} G_{S_{m, d, h}} R_{D n M C P_{m, d, h}} b_{R D n_{m, d, h}}
\end{aligned}
$$

The generation costs are considered from part (a) to (f) in (13). Part (a) accounts the fixed operation cost for electricity in month $m$, where $T_{F_{m}}$ is the regulated tariff fixed charge for electricity. Part (b) models the electricity purchased from the power utility, where $U_{L_{m, d, h}}$ is the electricity purchase at time $m, d$, and $h$, and $T_{E_{m, d, h}}$ is the regulated tariff for electricity. Part (c) relates the variable operation and maintenance costs of generation, where $G_{T_{j, m, d, h}}$ is the total power generated by technology $j, C_{V C_{j}}$ is the generation cost of technology $j$ during month $m$; and $C_{O_{O M V}}$ is the variable annual operation and maintenance costs of technology $j$. Afterwards, parts (d), (e) and (f) model the fixed annual operation and maintenance costs for MT/WT, PV and BA respectively. In these parts, $I_{G_{g}}$ is the number of units of generation technology $g$ installed; $P_{g}$ is the active power generated by the technology $g$; $C_{C C D_{g}}$ is the turnkey capital cost of generation technology $g ; A_{c}, A_{g}, A_{k}$ are the annuity factor for investments in technologies $c, g$ and $k ; C_{O M F_{c}}, C_{O M F_{g}}, C_{O M F_{k}}$ are the fixed annual operation and maintenance costs of technology $c, g$ and $k ; C_{F C C_{c, k}}$ is the fixed capital cost of generation technology $c$ or $k$; and $C a p_{c}$ and $C a p_{k}$ are the rated power capacity of generation technology $c$ and $k$.

Furthermore, the generation revenues are considered from part $(\mathrm{g})$ to $(\mathrm{k})$. Part $(\mathrm{g})$ is the revenue due to the exported power, where $G_{S_{m, d, h}}$ is the power generated to be exported at time $m, d$, and $h ; T_{E x_{m, d, h}}$ is the regulated tariff for electricity export at time $m, d$, and $h$; and $b_{T E x_{m, d, h}}$ is the binary decision of selling electricity to the stock at time $m$, $d$, and $h$. Parts (h), (i), (j) and (k) are the revenues due to AS supplying for spinning reserve, non-spinning reserve, and up- and down-frequency regulation respectively. In these parts, $S_{M C P_{m, d, h}}, N S_{M C P}, R_{U p M C P}$, and $R_{D n M C P}$ are the regulated tariff for the spinning reserve, non-spinnning reserve, and up- and down-frequency regulation, respectively, while the binary variables are described in Section 2.4 .

\subsection{Decision variables for enhanced POMMP methodology}

The decision variables are organized in the decision variables vector $\boldsymbol{x}$ and they are divided into two sets: $\left[x_{m t}, x_{w t}, x_{p v}, x_{b a}\right]$ with the capacities of the MT, WT, PV, and BA, respectivelly, and $\left[x_{b u s, m t}, x_{b u s, w t}, x_{b u s, p v}, x_{b u s, b a}\right]$ with the location of the the MT, WT, PV, and BA respectivelly [1]. It is important to highlight that in [1] the BA is located and sized based on the PV generation units. On the contrary, in the POMMP methodology, the decision variables vector considers a set of variables for the size and location of BA. This can be seen in (14):

$\boldsymbol{x}=\left[x_{m t}, x_{w t}, x_{p v}, x_{b a}, x_{b u s, m t}, x_{b u s, w t}, x_{b u s, p v}, x_{b u s_{b a}}\right]$

\subsection{Constraints functions for enhanced POMMP methodology}

For the proposed POMMP methodology, different constraints are adapted from [1, 3].

1) Energy balance in microgrid: the energy balance includes the generated, exported, imported, consumed power and losses in islanded and grid-connected modes in (15) and (16), where $T_{o P G_{m, d, h}}, P_{\text {Aexp }_{m, d, h}}$, $P_{\text {Aimp } m_{m, d, h}}, T_{o P D_{m, d, h}}$, and $P_{\text {loss }_{m, d, h}}$ are the total active power generated, exported, imported, demanded, and lost, respectively for the microgrid in grid-connected mode and in islanded mode. Additionally, the voltage $V$ limits at each node are constrained in (17). 


$$
\begin{aligned}
& T_{o P G_{m, d, h}}-P_{\text {Aexp }_{m, d, h}}+P_{\text {Aimp }_{m, d, h}} \\
& \quad=T_{o P D_{m, d, h}}+P_{\text {loss }_{m, d, h}} \quad \forall m, d, h \\
& T_{o P G I_{m, d, h}}=T_{o P D I_{m, d, h}}+P_{\text {lossI }_{m, d, h}} \quad \forall m, d, h \\
& V_{\min } \leq V_{N_{b}, m, d, h} \leq V_{\max } \quad \forall m, d, h
\end{aligned}
$$

2) Operation limits of distributed generators: the maximum and minimum operation limits of each DG, the maximum number of operation hours and the available physical area for distributed energy resources installation are considered in (18)-(20).

$$
\begin{aligned}
& \min \sum_{g} P_{g} \leq G_{T_{g, m, d, h}} \leq \max \sum_{g} P_{g} \quad \forall m, d, h \\
& \sum_{m} \sum_{d} \sum_{h} G_{T_{g, m, d, h}} \leq \max \sum_{g} P_{g} H_{g} \quad \forall m, d, h \\
& A U_{N_{t}, i} \leq A D i s p_{N_{t}, i} \quad \forall i: i \in\{P V, W T\}
\end{aligned}
$$

where $H_{g}$ is the number of hours that the units with technology $g$ can operate; $A U_{N t, i}$ is the used physical area at the bus $N_{t}$ by a technology $i$; and $A D i s p_{N_{t}, i}$ is the available physical area at the bus $N_{t}$ by a technology $i$. Note that the other variables are part of (13).

3) Operation limits of storage systems: as in the previous case, the operation limits of storage systems are constrained. Limits such as energy balance, maximum and minimum power flow capacity in a given node and the verification of the scheduled dispatch for the supply of AS are taken into account in (21)-(30).

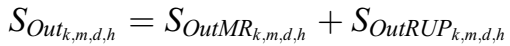

$$
\begin{aligned}
& +S_{\text {Out } S_{k, m, d, h}}+S_{\text {OutNS } S_{k, m, d, h}} \quad \forall k, m, d, h \\
& S_{I n_{k, m, d, h}}=S_{I n M R_{k, m, d, h}}+S_{I n R D n_{k, m, d, h}} \quad \forall k, m, d, h \\
& S O C_{k, m, d, h} \cdot C_{b a t_{k}}=S_{I_{k, m, d, h}}-S_{O u t_{k, m, d, h}} \\
& +S O C_{k, m, d, h-1} \cdot C_{b a t_{k}}\left(1-\varphi_{k}\right) \quad \forall k, m, d, h \\
& S O C_{k, m, d, 1}=S O C_{k, m, d, 24} \quad \forall k, m, d \\
& S O C_{k, m, d, h} \geq M S C_{k} \quad \forall k, m, d, h \\
& \operatorname{SOC}_{k, m, d, h} \leq 1 \quad \forall k, m, d, h \\
& S_{I n_{k, m, d, h}} \leq C_{b a t_{k}} \quad \forall k, m, d, h \\
& S_{\text {Out }_{k, m, d, h}} \leq C_{\text {bat }_{k}} \quad \forall k, m, d, h \\
& S_{I n_{k, m, d, h}} \leq b_{c h_{k, m, d, h}} M \quad \forall k, m, d, h \\
& S_{\text {Out }_{k, m, d, h}} \leq b_{d c h_{k, m, d, h}} M \quad \forall k, m, d, h
\end{aligned}
$$

where $S_{O u t_{k, m, d, h}}, S_{I_{n_{k, m, d, h}}}$, for the battery $k$, are the input and output power; $S_{I n M R_{k, m, d, h}}$ is the output of battery $k$ for the internal consumption of the microgrid; $S_{\text {Out }_{k, m, d, h}}$,
$S_{\text {Out NS }_{k, m, d, h}}, S_{\text {OutMR }_{k, m, d, h}}, S_{\text {OutRUP }_{k, m, d, h}}$ are the output power of battery $k$ for the spinning, non-spinning, and up- and downfrequency regulation, respectively; $\varphi_{k}$ is the losses due to decay or self-discharge in battery $k$; and $M$ is an arbitrary large number for binary considerations. Other variables linked with (21)-(30) are described in Section 2.2.

4) Export and import of energy to microgrid: it is guaranteed that there is not incoming and outgoing energy flow from and to the main grid at the same time in (31) and (32).

$U_{L_{m, d, h}} \leq b_{p s_{m, d, h}} M \quad \forall m, d, h$

$G_{S_{m, d, h}} \leq\left(1-b_{p s_{m, d, h}}\right) M \quad \forall m, d, h$

where $b_{p s_{m, d, h}}$ is the binary decision of selling or purchasing electricity to the main grid; $U_{L_{m, d, h}}$ is the electricity purchase; and $G_{S_{m, d, h}}$ is the power generated to be exported.

5) AS: the dispatches of AS are constrained in the microgrid by the scheduled dispatch, supplying time window, power capacity and a minimum bid to participate in each of the energy markets in (33)(44). Thus, the binary decisions for the AS supplying are verified along the horizon planning with (33) to (36).

$$
\begin{aligned}
& S_{m, d, h} \leq b_{S_{m, d, h}} M \\
& N S_{m, d, h} \leq b_{N S_{m, d, h}} M \\
& R_{U p_{m, d, h}} \leq b_{R U p_{m, d, h}} M \\
& R_{D n_{m, d, h}} \leq b_{R D n_{m, d, h}} M
\end{aligned}
$$

where $S_{m, d, h}, N S_{m, d, h}, R_{U p_{m, d, h}}, R_{D n_{m, d, h}}$ are the total active power generated for spinning reserve, non-spinning reserve, and up- and down-frequency regulation, respectively; and $b_{S_{m, d, h}}, b_{N S_{m, d, h}}, b_{R U p_{m, d, h}}, b_{R D n_{m, d, h}}$ are the binary decision of selling certain type of AS, respectively. The AS supplying time is constrained for each type from (37) to (40), where $h^{\prime}=\{h, h+1, . ., h+\theta\}$. For this purpose, $\theta$ represents the AS supplying time.

$$
\begin{aligned}
& \sum_{h^{\prime}} b_{S_{m, d, h}} \geq\left(b_{S_{m, d, h}}-b_{S_{m, d, h-1}}\right) \theta \\
& \sum_{h^{\prime}} b_{N S_{m, d, h}} \geq\left(b_{N S_{m, d, h}}-b_{N S_{m, d, h-1}}\right) \theta \\
& \sum_{h^{\prime}} b_{R U p_{m, d, h}} \geq\left(b_{R U p_{m, d, h}}-b_{R U p_{m, d, h-1}}\right) \theta \\
& \sum_{h^{\prime}} b_{R D n_{m, d, h}} \geq\left(b_{R D n_{m, d, h}}-b_{R D n_{m, d, h-1}}\right) \theta
\end{aligned}
$$

The constrains (41) to (43) are intended to guarantee a minimum capacity in the microgrid to assure its participation in the AS markets. 


$$
\begin{aligned}
& S_{m, d, h} \geq b_{S_{m, d, h}} S_{B i d}^{\min } \\
& N S_{m, d, h} \geq b_{N S_{m, d, h}} N S_{B i d}^{\min } \\
& R_{U p_{m, d, h}} \geq b_{R U p_{m, d, h}} R_{U p, B i d}^{\min } \\
& R_{D n_{m, d, h}} \geq b_{R D n_{m, d, h}} R_{D n, B i d}^{\min }
\end{aligned}
$$

where $S_{B i d}^{\min }, N S_{B i d}^{\min }, R_{U p, B i d}^{\min }$, and $R_{D n, B i d}^{\min }$ are the minimum bid for spinning and non-spinning reserve market, and up- and down-frequency regulation markets.

\subsection{True-multi-objective optimization and decision- making algorithms}

In this paper, the NSGA-II proposed in [25] has been used to solve the optimization problem and find the Paretooptimal solutions. On the other hand, to enhance the implementation of the planning methodology, a multi-criteria decision-making based on the AHP strategy is implemented as the last step to select a solution from the optimal Pareto-set based on the criteria of decision-maker $[26,27]$.

\subsubsection{Multi-objective optimization algorithm-NSGA-II}

NSGA-II has been amply used in the engineering field and is presented for the first time in [25]. For solving the problem with the algorithm, a certain number of individuals $p$ in the population and generations $g$ should be tuned.

\subsubsection{Multi-criteria decision-making algorithm-AHP}

In the AHP, pairs of criteria and possible solutions are compared based on a predefined relevance-level scale [27]. The problem is hierarchically discomposed and ranked, making the decision in a descendant order. The strategy implemented in this paper is a combination from [20, 28, 29] approaches as follow.

1) The decision-making is organized as a hierarchic structure with three levels as shown in Fig. 1. The main goal is in Level 1. In Level 2, the decisionmaking criteria are set as each one of the three objective functions. In Level 3, the alternatives that will be evaluated are established. For this purpose, each criteria has $p$ alternatives, which are each solution in the Pareto-set for each objective function, and are the output of the NSGA-II.

2) The objective functions (Level 2) are weighted by comparing in pairs based on the decision-maker

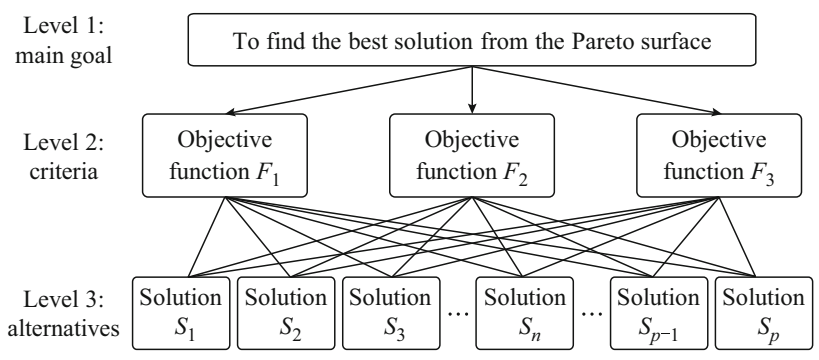

Fig. 1 Hierarchic structure used for the decision-making

criteria and the question "how relevant is the objective function with respect to the other?". For this purpose the Saaty's scale with 9 relevance levels is used [20]. This comparison gives rise to the comparison matrix $\boldsymbol{B}$ for $n=3$ objective functions (45).

$\boldsymbol{B}=\left[\begin{array}{cccc}1 & b_{12} & \cdots & b_{1 n} \\ b_{21} & 1 & \cdots & b_{2 n} \\ \vdots & \vdots & & \vdots \\ b_{n 1} & b_{n 2} & \cdots & 1\end{array}\right]$

where $b_{i j}$ is the weight for each pair of objective functions based on Saaty's scale and $b_{i j}=1 / b_{j i}$.

3) The consistency among the weights is reviewed with the maximum eigenvalue of $\boldsymbol{B}$ and the consistency index that is calculated and evaluated as in [20, 29].

4) The normalized relative relevance for each objective function are calculated based on the geometric mean (46):

$$
W_{i}=\frac{\left(\prod_{i=1}^{n} b_{i j}\right)^{\frac{1}{n}}}{\sum_{i=1}^{n}\left(\prod_{i=1}^{n} b_{i j}\right)^{\frac{1}{n}}}
$$

Then, the normalized weights give rise to the vector $\boldsymbol{W}_{F}$, with $n=3$ in this case (47):

$$
\boldsymbol{W}_{F}=\left[\begin{array}{llll}
W_{1} & W_{2} & \cdots & W_{n}
\end{array}\right]^{\mathrm{T}}
$$

5) The alternatives in Level 3 (solutions for each objective function) are weighted as in 2) following the Saaty's scale of 9 degrees. Thus, the solutions are organized in ascendant or descendant order depending on the maximisation or minimisation goal, and the Saaty's scale is assigned in this order. This gives rise to the comparison matrix $S_{p \times p}$ for $p$ equals to number of solutions. Afterwards, 3) and 4) are applied for $S$, which leads to a vector $\boldsymbol{W}_{S}$ for each objective function (48). 
$\boldsymbol{W}_{S}=\left[\begin{array}{llll}W_{S_{1}} & W_{S_{2}} & \cdots & W_{S_{p}}\end{array}\right]^{\mathrm{T}}$

6) The ranking of each solution $p$ of the Pareto-solutions is calculated with (49):

$$
\boldsymbol{R}_{p}=\left[\begin{array}{ccc}
F_{1} & F_{2} & F_{3} \\
W_{S_{1}} & W_{S_{1}} & W_{S_{1}} \\
W_{S_{2}} & W_{S_{2}} & W_{S_{2}} \\
\vdots & \vdots & \vdots \\
W_{S_{p}} & W_{S_{p}} & W_{S_{p}}
\end{array}\right]\left[\begin{array}{l}
W_{F_{1}} \\
W_{F_{2}} \\
W_{F_{3}}
\end{array}\right]
$$

7) Finally, the solution with the highest ranking is chosen from the optimal Pareto-set of solutions.

\section{Microgrids planning methodology}

The POMMP methodology is based on the methodology presented in [1], where $t$ is time segment, $p$ is individual of the population, $g$ is generation of the population. The enhanced methodology is presented in Fig. 2. Note that DER stands for distributed energy resources. The methodology can be read following the step indexes. In the methodology, an optimal power dispatch along the planning horizon is included, and the stochastic operation of the units is modelled with Monte Carlo simulation of the primary energy, based on PDFs of the wind speed and solar irradiation and historical time series in (2)-(4). Thus, a simulation of the network operator is executed (2)-(3) in order to determine the AS demand from the operator and then generate the decisions variables vector (6). Furthermore, the (9), (111) and (14) have been included to consider the improvements in the energy storage systems considerations, as well as the (8), which represent the binary C/D variables of the DS based on the market AS demand and the demand management approach described in Section 2.2. Finally, (18) introduces the multi-criteria decisionmaking approach implemented to choose the most convenient solution from the Pareto-front based on a decision maker's criteria and the AHP technique.

\section{Modelled cases and decision-making criteria}

In this paper, two modelled cases (with information of two real markets), and the test system PG\&E 69-bus are used to test the POMMP methodology. The test system used is presented and described in [1] as well as relevant features of the methodology proposed in [1] that are kept. For example, the multi-region capability for considering more than one area of wind-speed and solar irradiation.

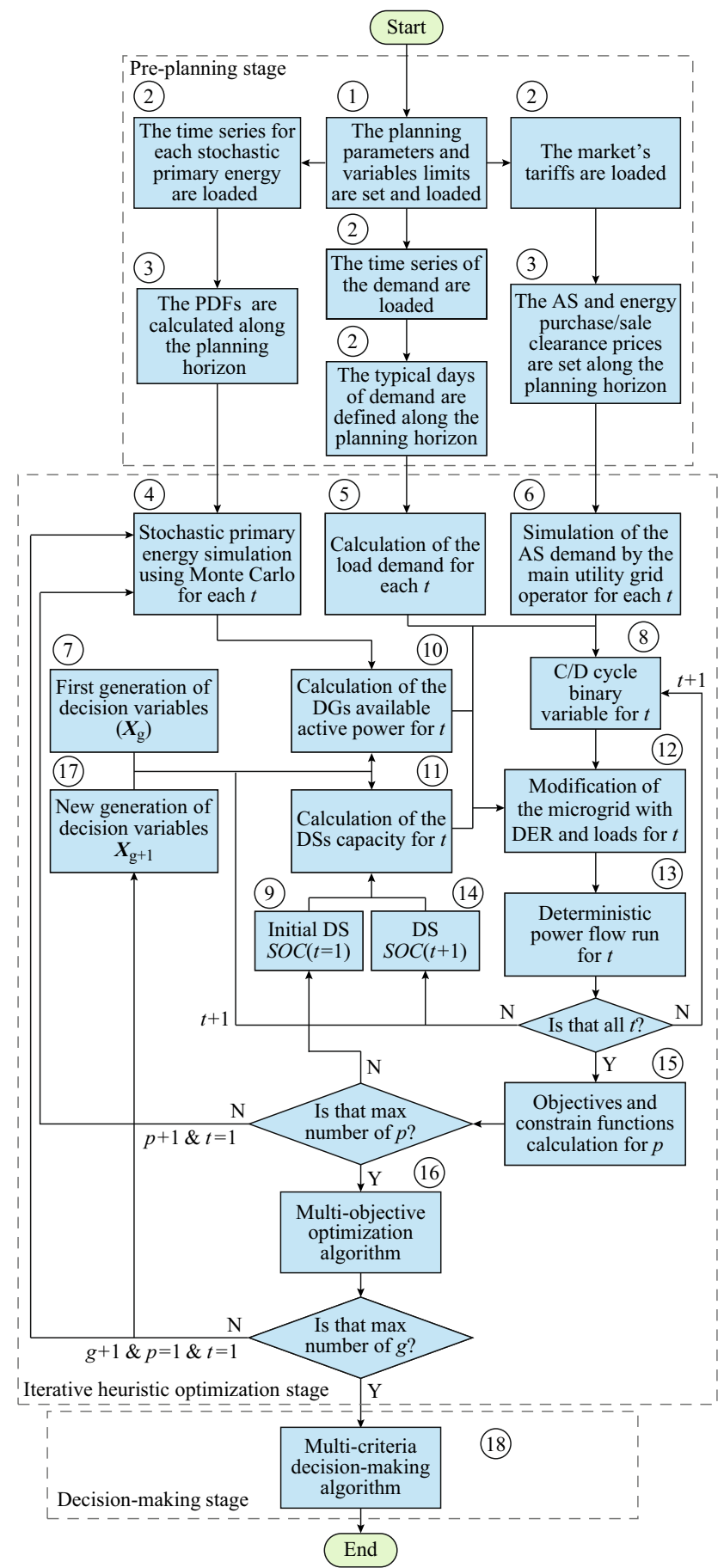

Fig. 2 Proposed POMMP methodology

Furthermore, the demand time-series for the three typical days used in the planning were adapted from [1, 30] as well as the time series for wind speed, solar irradiation that were taken from the German Meteorological Office (Deutscher Wetterdienst-DWD). The time series are processed and the Weibull and Log-normal PDF are defined to describe the wind speed and global solar irradiation, respectively. A PDF for each time-segment of one hour of each of the 
three typical days of a month (weekdays, weekends and peak days) were defined for both wind speed and solar irradiation areas, and the Monte Carlo simulation was set for 5000 aleatory values.

Other parameters such as the battery efficiency, $D O D$ and $M O D$, types of location in the microgrid's buses, installation area required by the distributed energy resources, voltage limits, and the NSGA-II code adaptation in MATLAB are kept as in [1]. Regarding the NSGA-II parameters tuning, the population and generations numbers are set up based on a specific tuning process as $p=1000$ individuals and $g=50$ generations for the two modelled cases.

To test the POMMP methodology, the rated power of the DG and DS technologies are chosen as $31 \mathrm{~kW}, 120 \mathrm{~kW}$, $100 \mathrm{~kW}$, and $600 \mathrm{~kW}$ for MT, WT, PV, and BA, respectively. The lower optimization boundaries are set up as zero for all the technologies, and the upper boundaries were defined as $310 \mathrm{~kW}$ (10 units) for MT, 1.2 MW (10 units) for WT, $100 \mathrm{~kW}$ for PV, and $600 \mathrm{~kW}$ for BA. The number of possible buses with distributed energy resources in the microgrid is set as a maximum 5 buses for MT, WT, and $\mathrm{BA}$; and maximum of 10 buses for PV. It is important to mention that the approach used in [1] regarding the discrete and continuos sizing of DG and DS was modified based on [3], where a PV and BA are sized as discrete due to their modularity and MT and WT as continuous variables. These limits are chosen with the aim to test the methodology, although they can be modified depending on the user requirements.

Two cases were implemented. Case 1 is POMMP for PJM market and load shedding in islanded operation. Case 2 is POMMP for CAISO market and load shedding in islanded operation. The load shedding strategy in islanded operation was adopted as in [1] to demonstrate an additional planning condition that can be considered with the POMMP methodology.

\subsection{Economical parameter and market description}

For this paper, the technical and economic data used to describe all the distribution energy resources are obtained from $[1,31,32]$. The specific DG and DS parameters chosen such as $C_{C C D}, C_{O M V}, C_{O M F}, C_{F C C}, \eta_{c h}, \eta_{d c h}, D O D$, $M S C$ and $\eta_{b a}$ can be consulted in [38] for purposes of result reproducibility.

Regarding the market data, the cities of San Francisco and Baltimore belonging to the territory of CAISO and PJM respectively are selected to implement the model in locations with different AS rates and market prices as in [3]. The tariff information used is based on data available in [31]. Furthermore, the PG \& E and Baltimore Gas \& Electric (BG \& E) generation rates are selected for the cities of San Francisco and Baltimore respectively from [3], and the transmission rates considered in [33] are added.

The time series of the tariff for exporting energy to the main utility grid $(T E x)$ is based on the clearing price of the market. The data are obtained for 2014 from [34] for CAISO market and from [35] for PJM market.

The tariffs for the AS provision are chosen from 2014 historical time series data of the clearing prices of CAISO and PJM markets [36, 37]. It must be considered that these values correspond to the average price of each day-type planned along the planning horizon.

All the market data used for simulations can also be consulted in [38].

\subsection{Decision-making criteria}

For the solution of the POMMP methodology, the authors take the role of decision-makers in order to apply the multi-criteria decision-making approach described before. Therefore, the objective function relevance order has been defined by the authors for the methodology test as:

1) $F_{1}$ : residual active power in grid-connected mode.

2) $F_{3}$ : operation cost of the microgrid in grid-connected mode.

3) $F_{2}$ : mismatch power in islanded mode.

Following the Saaty's scale in [20], the comparison matrix for the objective functions that has been defined by the decision-maker for the two study cases is shown in (50). Thus, the intensity of importance 1 (equal importance) is for two criteria (objective functions) that contribute equally to the main objective of the AHP; 3 (moderate importance) is for an experience and judgment slightly favor one criteria over another; and 5 (strong importance) is for an experience and judgment that strongly favors one criteria over another.

$$
\begin{aligned}
& \begin{array}{lll}
F_{1} & F_{2} & F_{3}
\end{array} \\
& \boldsymbol{B}=\begin{array}{l}
F_{1} \\
F_{2} \\
F_{3}
\end{array}\left[\begin{array}{lll}
b_{11} & b_{12} & b_{13} \\
b_{21} & b_{22} & b_{23} \\
b_{31} & b_{32} & b_{33}
\end{array}\right]=\begin{array}{l}
F_{1} \\
F_{2} \\
F_{3}
\end{array}\left[\begin{array}{ccc}
1 & 5 & 3 \\
1 / 5 & 1 & 3 \\
1 / 3 & 1 / 3 & 1
\end{array}\right]
\end{aligned}
$$

The comparison matrix for the Pareto-set of solutions (alternatives) has been built assigning all 9 values of the Saaty's scale and solving the procedure described in Section 3.4.2. Note that the objective relevance order can be modified based on the other preferences and/or requirements. 


\section{Simulation results and discussion}

\subsection{Case 1: POMMP for PJM market and load shedding}

The Pareto-optimal results of the POMMP under the PJM market conditions are presented in Fig. 3, where each point (individual $p$ ) represents a possible optimal solution for the planning problem in the objective functions space, and $\mathrm{S}$ stands for the solution. Solutions show a range of possible microgrid designs, whose operation cost can increase from $\$ 0.5$ million up to $\$ 1.1$ million, with a residual capacity for the AS provision and market participation up to $1 \mathrm{MW}$. It can also be analyzed that the mismatch power in islanded mode can also be considered as a design criteria if it is set up as a priority criteria in order to reduce the possible operation problems due to an installed overcapacity.

In Fig. 3, S41 is the solution of the minimum operation cost, and S74 and S3 are the first and second best rankedsolutions from the AHP decision-making strategy outcome. The objective function results for each solution are presented in Table 1 .

Table 1 shows that between these two solutions, an increase of $40 \mathrm{~kW}$ in the residual power leads to an increase of $\$ 54300$ in the operation of the microgrid, which should be considered. These results support the advantages of the POMMP methodology with a true-multi-objective approach because it is possible to analyze the possible trade-off options. Furthermore, from the POMMP methodology outcome, it can be seen that a minimum operation cost can be achieved for the extreme S41 with $\$ 8690400$ that is slightly bigger than the $50 \%$ of the generation cost for S74. However, there is no available power capacity for the AS provision. With the multi-objective approach, it is possible to find a convenient trade-off among the objective functions, with an improvement in the reserve capacity for the AS supplying up to $1011.8 \mathrm{~kW}$ with the S3.

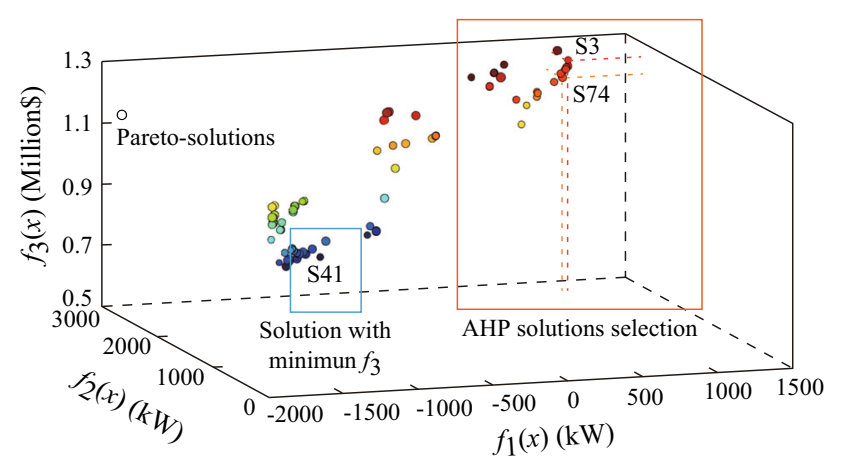

Table 1 Optimal solutions for microgrid planning in Case 1

\begin{tabular}{llll}
\hline Solution & $f_{1}(x)(\mathrm{kW})$ & $f_{2}(x)(\mathrm{kW})$ & $f_{3}(x)(\$)$ \\
\hline S74 & 972.0 & 2722.0 & 1193700 \\
S3 & 1011.8 & 2733.5 & 1248000 \\
\hline
\end{tabular}

Operational characteristics and market participation capabilities such as the total energy exported, imported, generated, demanded, revenues and expenses purchase of the two best solutions chosen by AHP for the participation of microgrid in the PJM electricity market, are shown in the Table 2.

Table 2 shows that, despite the generated, demanded and imported energies are relatively similar in terms of $\mathrm{MWh} /$ year, the energy purchasing cost will increase $3.15 \%$ for S3. Therefore, it can be claimed that the POMMP can offer a set of possible solutions with small technical variations that can give rise to optimal extra savings in the microgrid operation.

The best solution in terms of the multicriteria decisionmaking AHP is S74. The optimal sizing and location of distributed energy resources for this solution are presented in Table 3.

Graphically, the PG\&E distribution network transformed as a microgrid is shown in Fig. 4.

Comparing it with the results of [1], Fig. 4 shows that the POMMP methodology gives a set of independently optimally sized and located BA units, which together with the demand management approach can offer a better flexibility to the microgrid AS provision.

\subsection{Case 2: POMMP for CAISO market and load shedding in islanded operation}

Now, the POMMP methodology is used to plan a microgrid under the market condition of CAISO. The optimal Pareto-set of solutions is shown in Fig. 5.

Table 2 Operational characteristics and market participation for S74 and $\mathrm{S} 3$

\begin{tabular}{lll}
\hline Output parameter & S74 & S3 \\
\hline Total energy generated (MWh/year) & 2984.4 & 3006.5 \\
Total energy demanded (MWh/year) & 2268.2 & 2276.6 \\
Total energy imported (MWh/year) & 29.47 & 29.82 \\
Total energy exported (MWh/year) & 680.59 & 691.78 \\
Income from export of energy (\$/year) & 4283.2 & 4364.7 \\
Income from AS provision (\$/year) & 9348.3 & 9534.5 \\
Expenses from energy purchasing (\$/year) & 20047 & 20679 \\
\hline
\end{tabular}

Fig. 3 Optimal Pareto-solutions for microgrid planning in Case 1 
Table 3 Optimal capacities DG and DS units for S74

\begin{tabular}{|c|c|c|c|}
\hline Type & Location bus & No. of units & Capacity $(\mathrm{kW})$ \\
\hline \multirow[t]{4}{*}{ MT } & 54 & 10 & 212.7 \\
\hline & 51 & 6 & 127.6 \\
\hline & 39 & 2 & 42.5 \\
\hline & 3 & 6 & 127.6 \\
\hline \multirow[t]{5}{*}{ WT } & 69 & 8 & 960 \\
\hline & 53 & 8 & 960 \\
\hline & 68 & 10 & 1200 \\
\hline & 64 & 3 & 360 \\
\hline & 9 & 8 & 960 \\
\hline \multirow[t]{10}{*}{ PVs } & 42 & - & 32.8 \\
\hline & 8 & - & 72.9 \\
\hline & 26 & - & 78.9 \\
\hline & 35 & - & 4.7 \\
\hline & 34 & - & 91.5 \\
\hline & 2 & - & 31.4 \\
\hline & 37 & - & 74.4 \\
\hline & 23 & - & 22.5 \\
\hline & 19 & - & 34.4 \\
\hline & 58 & - & 2.0 \\
\hline \multirow[t]{5}{*}{ BA } & 22 & - & 481.1 \\
\hline & 65 & - & 316.3 \\
\hline & 41 & - & 108.5 \\
\hline & 10 & - & 170.3 \\
\hline & 12 & - & 221.4 \\
\hline
\end{tabular}

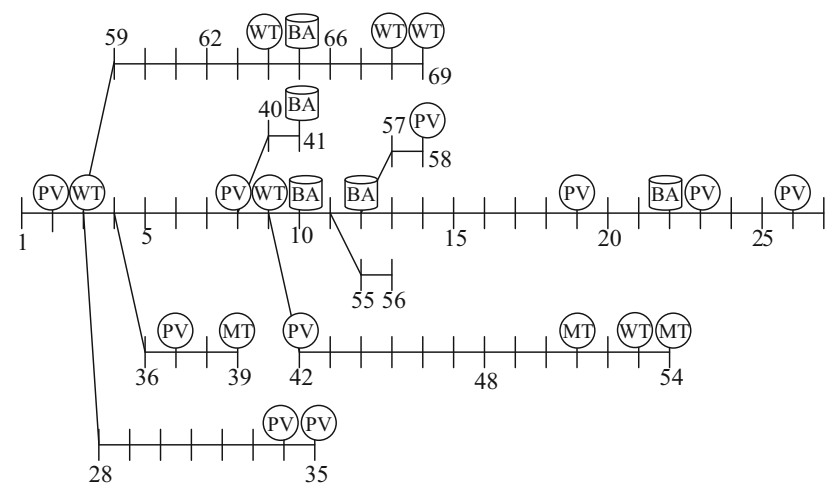

Fig. 4 Optimal microgrid with 69 buses

Comparing the result of the two cases, it can be analyzed that, despite that the operation cost keeps relatively between the same limits, the residual power capacity of the microgrid to offer AS for the case of the CAISO market is lower than the PJM case. When the AHP multi-criteriadecision-making technique was applied, it was found that the two most convenient decisions are the solution S61 and S96. The results are shown in Table 4.

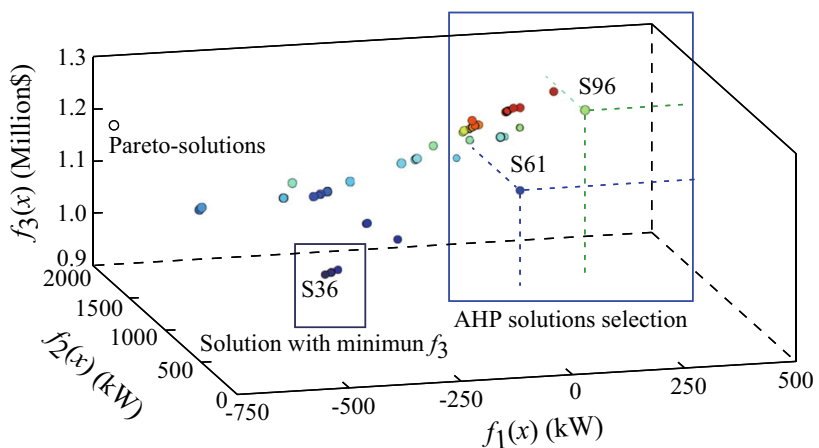

Fig. 5 Optimal Pareto-solutions for microgrid planning in Case 2

Table 4 Optimal solutions for microgrid planning in Case 2

\begin{tabular}{llll}
\hline Solution & $f_{1}(x)(\mathrm{kW})$ & $f_{2}(x)(\mathrm{kW})$ & $f_{3}(x) \$$ \\
\hline S61 & 100.1 & 1348.2 & 1081500 \\
S96 & 267 & 1748.5 & 1208000 \\
\hline
\end{tabular}

Furthermore, it can be seen that a minimum operation cost can be achieved for the extreme S36 with $\$ 943000$, which is smaller than the generation cost for S61 in $\$ 138500$. However, there is no available power capacity for the AS provision. Using the multi-objective approach, it is possible to find a solution with an improvement in the reserve capacity for the AS supplying up to $267 \mathrm{~kW}$ with S96.

The operation characteristics for the two solutions are shown in Table 5. The size and location of the distributed energy resources for the optimal design in S61 are described in Table 6.

From the results, it is found that the installed capacity of distributed energy resources is lower for Case 2 in comparison with Case 1. For example, the total energy generated by the microgrid in Case 2 for the solution S61 is the $58 \%$ of the energy generated by the microgrid in Case 1 for the solution S71. Furthermore, the incomes from the AS provision are only $21 \%$ of the income achieved in Case 1 .

Table 5 Operational characteristics and market participation for S61 and $\mathrm{S} 96$

\begin{tabular}{lll}
\hline Output parameter & S61 & S96 \\
\hline Total energy generated (MWh/year) & 1743.3 & 1906.6 \\
Total energy demanded (MWh/year) & 2175.8 & 2229.1 \\
Total energy imported (MWh/year) & 538.76 & 463 \\
Total energy exported (MWh/year) & 40.4 & 64.49 \\
Income from export of energy (\$/year) & 760.8 & 1742.3 \\
Income from AS provision (\$/year) & 1971.7 & 2807.5 \\
Expenses from energy purchasing (\$/year) & 57185 & 49048 \\
\hline
\end{tabular}


Table 6 Optimal DG and DS units capacity for solution S61

\begin{tabular}{|c|c|c|c|}
\hline Type & Location bus & No. of units & Capacity $(\mathrm{kW})$ \\
\hline \multirow[t]{5}{*}{ MT } & 38 & 4 & 85.1 \\
\hline & 37 & 2 & 42.5 \\
\hline & 63 & 8 & 170.2 \\
\hline & 11 & 2 & 42.5 \\
\hline & - & - & - \\
\hline \multirow[t]{5}{*}{ WT } & 9 & 6 & 720 \\
\hline & 31 & 6 & 720 \\
\hline & 65 & 5 & 600 \\
\hline & 62 & 1 & 120 \\
\hline & 27 & 5 & 600 \\
\hline \multirow[t]{10}{*}{ PV } & 25 & - & 74.1 \\
\hline & 49 & - & 62 \\
\hline & 26 & - & 32.5 \\
\hline & 68 & - & 89.5 \\
\hline & 40 & - & 6.1 \\
\hline & 53 & - & 47.7 \\
\hline & 54 & - & 93.2 \\
\hline & 46 & - & 41.5 \\
\hline & 23 & - & 81.6 \\
\hline & - & - & - \\
\hline \multirow[t]{5}{*}{ BA } & 14 & - & 188.7 \\
\hline & 30 & - & 507.4 \\
\hline & 43 & - & 491.4 \\
\hline & 8 & - & 528.8 \\
\hline & 5 & - & 441.1 \\
\hline
\end{tabular}

\section{Conclusion}

In this paper, POMMP with three different objective functions has been proposed in order to enhance the AS provision to the main utility grid. The methodology is formulated and solved as a true-multi-objective optimization problem, and a multi-criteria decision-making strategy based on the AHP technique is implemented. In POMMP, the DS size and location have been defined as individual decision variables, a C/D approach based on the AS provision demand is proposed, and a demand management strategy is formulated. It is corroborated with the results that POMMP considers the AS market aspects, showing the relevance of considering the market conditions as part of a true-multi-objective approach. For example, CAISO market offers lower tariffs and conditions for an open AS provision market compared to the PJM market. Then, the POMMP methodology plans the microgrid for the CAISO case with an optimal installed capacity $58 \%$ lower than the one of the optimal solution of the PJM case. Furthermore, POMMP methodology can consider other demand management techniques such as load shedding in islanded operation mode. Future research will be related to the optimal planning of resources under the same methodology to supply other types of AS such as voltage regulation. Furthermore, a wide range of technical questions about the stability of microgrid and main grid from the planning step will lead to future research for extending flexible planning methodologies such as POMMP. This research should consider a greater interaction of the microgrid with the main grid. Finally, it is expected that methodologies as POMMP can be tested under different market conditions, networks and study cases in order to identify open market features that can benefit the revenue streams of microgrid and support their implementation.

Acknowledgements We would like to thank the program "Becas para Doctorados Nacionales 2014 - 647" of Colciencias as well as the students Andres Felipe Penaranda Bayona and Pablo Elver Mosquera Duarte from Universidad Nacional de Colombia.

Open Access This article is distributed under the terms of the Creative Commons Attribution 4.0 International License (http:// creativecommons.org/licenses/by/4.0/), which permits unrestricted use, distribution, and reproduction in any medium, provided you give appropriate credit to the original author(s) and the source, provide a link to the Creative Commons license, and indicate if changes were made.

\section{References}

[1] Contreras SF, Cortes CA, Myrzik JM (2018) Multi-objective probabilistic power resources planning for microgrids with ancillary services capacity. In: Proceedings of the 2018 IEEE power systems computation conference (PSCC), Dublin, Ireland, 11-15 June 2018, 8 pp

[2] Chowdhury S, Crossley P (2009) Microgrids and active distribution networks. The Institution of Engineering and Technology, London

[3] Cardoso G, Stadler M, Mashayekh S et al (2017) The impact of ancillary services in optimal DER investment decisions. Energy 130:99-112

[4] Xiang Y, Liu J, Li F et al (2016) Optimal active distribution network planning: a review. Electr Power Compon Syst 44 (10):1075-1094

[5] Georgilakis PS, Hatziargyriou ND (2015) A review of power distribution planning in the modern power systems era: models, methods and future research. Electr Power Compon Syst 121:89-100

[6] Gamarra C, Guerrero JM (2015) Computational optimization techniques applied to microgrids planning: a review. Renew Sustain Energy Rev 48:413-424

[7] Yuen C, Oudalov A (2007) The feasibility and profitability of ancillary services provision from multi-microGrids. In: Proceedings of the 2007 IEEE Lausanne powertech, Lausanne, Switzerland, 1-5 July 2007, 6 pp

[8] Alarcon-Rodriguez A, Ault G, Galloway S (2010) Multi-objective planning of distributed energy resources: a review of the state-of-the-art. Renew Sustain Energy Rev 14(5):1353-1366 
[9] Arefifar SA, Mohamed YR, El-Fouly THM (2012) Supply-adequacy-based optimal construction of microgrids in smart distribution systems. IEEE Trans Smart Grid 3(3):1491-1502

[10] Arefifar SA, Mohamed YAI (2014) Probabilistic optimal reactive power planning in distribution systems with renewable resources in grid-connected and islanded modes. IEEE Trans Ind Electron 61(11):5830-5839

[11] Zidan A, Shaaban MF, El-Saadany EF (2013) Long term multiobjective distribution network planning by DG allocation and feeders reconfiguration. Electr Power Syst Res 105:95-104

[12] Guo L, Wang N, Lu H et al (2016) Multi-objective optimal planning of the stand-alone microgrid system based on different benefit subjects. Energy 116:353-363

[13] Guo L, Liu W, Jiao B et al (2014) Multi-objective stochastic optimal planning method for stand-alone microgrid system. IET Gener Transm Distrib 8(7):1263-1273

[14] Gomes MH, Saraiva JT (2010) Allocation of reactive power support, active loss balancing and demand interruption ancillary services in microgrids. Electr Power Syst Res 80(10):1267-1276

[15] Madureira A, Pecas LJ (2012) Ancillary services market framework for voltage control in distribution networks with microgrids. Electr Power Syst Res 86:1-7

[16] Lee YD, Jiang JL, Su HJ et al (2016) Ancillary voltage control for a distribution feeder by using energy storage system in microgrid. In: Proceedings of the 2016 IEEE 7th international symposium on power electronics for distributed generation systems (PEDG), Vancouver, Canada, 27-30 June 2016, 7 pp

[17] Majzoobi A, Khodaei A (2017) Application of microgrids in providing ancillary services to the utility grid. Energy 123:555563

[18] Zhou Y, Panteli M, Moreno R et al (2018) System-level assessment of reliability and resilience provision from microgrids. Appl Energy 230:374-392

[19] Husein M, Chung IY (2018) Optimal design and financial feasibility of a university campus microgrid considering renewable energy incentives. Appl Energy 225(2):273-289

[20] Saaty TL (2008) Decision making with the analytic hierarchy process. Int J Serv Sci 1(1):83-98

[21] Banshwar A, Sharma NK, Sood YR et al (2016) An investigation of the various approaches for competitive procurement of ancillary services in deregulated power sector. In: Proceedings of the 2016 IEEE international conference on electrical power and energy systems (ICEPES), Bhopal, India, 14-16 December 2016, 6 pp

[22] Kyriakides E, Suryanarayanan S, Vittal V (2015) Electric power engineering research and education. Power electronics and power systems. Springer International Publishing, Cham

[23] Scholz D, Musgens F (2017) How to improve standard load profiles: updating, regionalization and smart meter data. In: Proceedings of the 14th international conference on the European energy market, Dresden, Germany, 6-9 June 2017

[24] Branke J, Deb K, Miettinen K et al (2008) Multiobjective optimization: interactive and evolutionary approaches. Springer, Berlin

[25] Deb K, Pratap A, Agarwal S et al (2002) A fast and elitist multiobjective genetic algorithm: NSGAII. IEEE Trans Evol Comput 6(2):182-197

[26] Kumar A, Sah B, Singh AR et al (2017) A review of multi criteria decision making (MCDM) towards sustainable renewable energy development. Renew Sustain Energy Rev 69:596609

[27] De Brito MM, Evers M (2016) Multi-criteria decisionmaking for flood risk management: a survey of the current state of the art. Nat Hazards Earth Syst Sci 16(4):1019-1033

[28] Ayadi O, Felfel H, Masmoudi F (2017) Analytic hierarchy process-based approach for selecting a Pareto-optimal solution of a multi-objective, multisite supply-chain planning problem. Eng Optim 49(7):1264-1280

[29] Alonso JA, Lamata MT (2006) Consistency in the analytic hierarchy process: a new approach. Int J Uncertain Fuzziness Knowl Based Syst 14(4):445-459

[30] Subcommittee P (1979) IEEE reliability test system. IEEE Trans Power Appar Syst 98(6):2047-2054

[31] Stadler M (2008) DER-CAM (distributed energy resources customer adoption model) Lawrence Berkeley National Laboratory (LBNL). https://building-microgrid.lbl.gov/projects/dercam. Accessed 30 September 2018

[32] IRENA (2014) International renewable energy agency (IRENA). https://www.irena.org/. Accessed 30 September 2018

[33] Yura JK (2014) Electric schedule E-19-medium general demand-metered TOU service. https://www.pge.com/. Accessed 30 September 2018

[34] Consulting L (2014) CAISO (California ISO): day-ahead price. http://www.energyonline.com/Data/GenericData.aspx?DataId= 22\&CAISO__Day-Ahead_Price. Accessed 30 September 2018

[35] PJM (2014) Hourly real-time and day-ahead LMP. http://www. pjm.com/markets-and-operations/energy/real-time/monthlylmp. aspx. Accessed 30 September 2018

[36] OASIS (2014) California ISO (CAISO), OASIS, AS clearing prices. http://oasis.caiso.com/mrioasis/logon.do. Accessed 30 September 2018

[37] PJM (2014) Ancillary service. http://www.pjm.com/markets-andoperations/ancillary-services.aspx. Accessed 30 September 2018

[38] Contreras SF (2018) POMMP methodology economical parameters and markets description for the study cases. https:// tinyurl.com/POMMP-Methodology. Accessed 30 September 2018

Sergio F. CONTRERAS received the bachelor and M.E. degrees from the Universidad Nacional de Colombia in 2010 and 2015, respectively. He is currently a Ph.D. student of the Universidad Nacional de Colombia and Researcher of the Institute for Automation Technologies, Institute for Automation Technology (IAT) of the University of Bremen, Germany. His research interests include the optimal planning and operation of active distribution networks for the integration of DERs, ancillary services supplying and modern power systems stability issues.

Camilo A. CORTES received the B.E. degree from the Universidad Nacional de Colombia in 2000 and the Ph.D. degree from the UNSJ, Argentina, in 2005. Since 2008, he has been an Associate Professor of the Universidad Nacional de Colombia, Bogot Campus. From 2015 to 2016, he was a visiting researcher at the Galvin Center for Electricity Innovation, Illinois Institute of Technology, USA. Since 2017 he is a German Academic Exchange Service Research Ambassador. His research interests focus on signal processing techniques, heuristic optimization, energy storage applications, microgrids planning, and modernization of power systems.

Johana M.A. MYRZIK received her master and Ph.D. degrees in 1992 and 2000, respectively. From 2000 to 2009, she was assistant/ associate professor in the field of power systems, urban supply systems and power quality at the Eindhoven University of Technology in the Netherlands. In 2009 she joined the University TU Dortmund, Germany, as full professor for energy efficiency and renewable energy resources. Since March 2018 Mrs. Myrzik is full professor for automation technology for energy systems at the University Bremen. Her fields of interest are integration of renewable energy sources, power quality, power system infrastructures, modeling and control. 